\title{
Valence Focus and the Perception of Facial Affect
}

\author{
Lisa Feldman Barrett \\ Boston College
}

\author{
Paula M. Niedenthal \\ National Center for Scientific Research and \\ University of Clermont-Ferrand
}

\begin{abstract}
Individuals differ in the extent to which they emphasize feelings of pleasure or displeasure in their verbal reports of emotional experience, an individual difference termed valence focus (VF). This multimethod study indicates that VF is linked to heightened efficiency in perceptual processing of affective stimuli. Individuals higher in VF (i.e., who emphasized feelings of pleasure/displeasure in reports of emotional experiences) were more sensitive to changes in negative facial expressions than individuals lower in VF. The effect was not accounted for by current affective state or other personality characteristics. Implications for the validity of self-reported experienced emotion are discussed.
\end{abstract}

Valence is a basic property of affect (Russell \& Feldman Barrett, 1999). When reporting their experience, individuals differ in the extent to which they emphasize this property, reflecting individual differences in valence focus (VF; Feldman, 1995; Feldman Barrett, 1998, 2004). For example, the word tired, defined as unpleasant and low in arousal, can be used to communicate feeling sleepy (emphasizing low arousal), annoyed and miserable (emphasizing the unpleasantness), or fatigued (emphasizing both low arousal and displeasure). An individual high in VF uses emotion adjectives primarily to communicate pleasure and displeasure, whereas someone low in VF does this less so and uses emotion adjectives to communicate other properties of feelings as well. The measurement of VF is implicit because participants are not asked directly about their attention to pleasure and displeasure. Rather, $V F$ is defined as the extent to

Lisa Feldman Barrett, Department of Psychology, Boston College; Paula M. Niedenthal, National Center for Scientific Research and Department of Psychology, University of Clermont-Ferrand, Clermont-Ferrand, France.

Preparation of this article was supported by National Science Foundation Grants SBR 9727896 and BCS 0074688 and National Institute of Mental Health Grant K02 MH001981 awarded to Lisa Feldman Barrett. We thank Eliza Bliss-Moreau, Ana Hristic, Kirsten Lebo, Michele Tugade, and Michael Suvak for their help in collecting the data used in the report.

Correspondence concerning this article should be addressed to Lisa Feldman Barrett, Department of Psychology, Boston College, 427 McGuinn Hall, Chestnut Hill, MA 02467. E-mail: barrettli@bc.edu which participants focus on the pleasant or unpleasant property of the adjectives when making self-reports of experienced emotion.

At a conceptual level, it is assumed that differential emphasis on valence during the self-report process reflects differential experience of pleasure and displeasure. A reasonable hypothesis is that people differ in their sensitivity to valenced information in the environment and, as a result, differ in the intensity and frequency of experienced pleasure and displeasure. Differential experience leads to differential word use, resulting in $\mathrm{VF}$.

\section{Perceptual Sensitivity to Valenced Information}

We examined the link between VF and perceptual sensitivity to valenced information by relying on a "morph movies" task (Niedenthal, Brauer, Halberstadt, \& Innes-Ker, 2001; Niedenthal, Brauer, Robin, \& Innes-Ker, 2002; Niedenthal, Halberstadt, Margolin, \& Innes-Ker, 2000). In the present version of the task, participants played 100-frame computerized movies in which a face displaying a neutral expression gradually changed to display a clear emotional expression (either angry, sad, or happy). The participants used a computerized mouse to control the speed with which each movie played and to stop each movie at the point where, for the first time, an emotional expression appeared on the face (i.e., the onset point).

The morph movies task was useful for studying the link between VF and perceptual sensitivity to valenced cues because it allowed a precise estimate of processing efficiency for pleasant and unpleasant information. For a given movie, the start point was a 
neutral face, and the end point was an objectively defined emotional expression (meaning that the emotional properties attributed to each face were validated by an independent group of respondents; see the Method section). All intervening movie frames were morphed from both the neutral and emotional faces. As the movie progressed, each frame contained an increasing amount of information from the emotional face (and less from the neutral face), and that information was available to be detected by the participant. The judged onset point was the point at which the participant detected the information. For a given movie, no label for the expression is given. The signal (i.e., the valenced information in the facial expression) gradually increased in intensity over the course of the movie. The task was to detect the earliest presence of that signal.

The morph movies task was also an ecologically valid way of assessing the VF-perceptual sensitivity link. People often see expressions emerging on the faces of others in everyday interactions. Although people may not often see an expression emerge smoothly from the face, or be able to control its emergence, they do often see traces of expressions on the faces of other individuals (such as those present in the morph movies). The task is thus a technique with which to address questions about individual differences in the efficiency of perceiving positive and negative facial expressions.

\section{Overview of the Present Study}

In this study, participants reported on their emotional experience over an extended experiencesampling period and also completed similarity ratings of emotion words, which allowed us to estimate their cognitive structure of emotion language. By comparing the two, we computed an index of VF for each individual. Two weeks after the experience-sampling period had ended, all participants performed the morph movies task. We hypothesized that individuals high in VF would perceive the onset of happy, angry, and sad facial expressions earlier than would those who are lower in VF, indicating their heightened sensitivity to valenced information on the face.

\section{Method}

\section{Participants}

Participants were 90 undergraduate students at Boston College ( 38 men) and ranged in age from 17 to 22 years $(M=19.00, S D=1.18) .{ }^{1}$ They were paid a total of $\$ 115$ and partook in several lotteries for their participation in all procedures. ${ }^{2}$

\section{Materials}

These data were taken from a large experiencesampling study of experienced emotion. Only those measures that are relevant to this report are presented here.

Experience-sampling procedure. The computerized experience-sampling procedure relied on palmtop computers that ran on custom software (Experience Sampling Program [ESP]; Barrett \& Feldman Barrett, 1999). Participants were beeped randomly 10 times per day for 28 days and were questioned about their momentary affective experience using 29 emotion-related terms. Affect terms were presented in a random order on each trial. Participants used 7-point Likert-type scales $(0=$ not at all, $3=$ a moderate amount, $6=$ a great deal) and indicated their ratings as quickly and accurately as possible by pressing numbers on the keyboard of the palm-top computer. If participants did not respond to the first prompt, they were beeped again several minutes later. If they failed to respond to the second prompt, then the trial was recorded as missing data. Ratings of experience and latencies were recorded.

Of the 29 terms used, 16 emotion-related adjectives sampling all parts of affective space were used to compute VF (enthusiastic, peppy, happy, satisfied, calm, relaxed, quiet, still, sleepy, sluggish, sad, disappointed, nervous, afraid, surprised, and aroused; see Feldman, 1995; Feldman Barrett, 1998, for details). The number of usable observation moments ranged substantially from 74 to 375 , with a mean of $181.37(S D=41.83)$.

\footnotetext{
${ }^{1}$ One hundred twenty-nine participants completed the experience-sampling portion of the study. Participants who completed the morph movie (and are included in this report) did not differ from those who did not complete that task on any demographic or emotional characteristics measured in this study.

${ }^{2}$ Some of the self-report and similarity rating data reported in this article have been used in a previous analysis. They were used to examine the link between arousal focus (or the tendency to emphasize felt activation or deactivation in verbal reports of emotional experience) and interoceptive sensitivity (Feldman Barrett, Quigley, Bliss-Moreau, \& Aronson, in press). As such, the hypotheses tested in that article do not in any way overlap with those presented here.
} 
Personality ratings. For the purposes of discriminant validity, participants completed a battery of questionnaires that included the Need For Evaluation Scale (Jarvis \& Petty, 1996), the Big Five Inventory (John, Donahue, \& Kentle, 1991), to measure neuroti$\operatorname{cism}(\mathrm{N})$ and extraversion $(\mathrm{E})$, and the behavioral inhibition system (BIS)/behavioral activation system (BAS) inventory (Carver \& White, 1994), to assess levels of activation in the BIS and BAS. Items for each scale were averaged. Only scores for $\mathrm{N}(M=$ $2.94, S D=0.82$, range $=1.13-4.50)$, E $(M=3.57$, $S D=0.83$, range $=1.63-5.00)$, BAS-Reward Responsiveness $(M=3.50, S D=0.37$, range $=2.60$ $4.00)$, and BIS $(M=3.02, S D=0.63$, range $=$ 1.43-4.00) were examined in this report. ${ }^{3}$ Need For Evaluation scores ranged from 2.38 to 3.81 , with a mean of $3.04(S D=0.30)$.

Similarity ratings. Participants performed a similarity judgment task that was implemented using SuperLab (1999) for a Macintosh platform. Participants were seated in front of a Macintosh computer and judged the similarity between all pairs of the 16 emotion-related adjectives of interest by pressing numbers on the computer keyboard corresponding to 7-point Likert-type scales $(1=$ extremely dissimilar, $4=$ unrelated, $7=$ extremely similar). Pairs were presented in a different random order for each participant, with an intertrial interval of $1,000 \mathrm{~ms}$.

Morph movies. Pictures of 10 happy, sad, angry, and neutral faces of male and female actor-models were selected for use in the morph movies task. These images are part of a larger set of photographs pretested on a group of 83 participants who established the validity of the actors' facial expressions (e.g., Halberstadt \& Niedenthal, 1997; Niedenthal et al., 2000). The face of the same individual did not necessarily appear in each facial expression category. Pretesting revealed that the selected faces displayed good examples of happy, sad, and angry expressions. On 1-7 scales, respectively, pretest participants provided an average happiness rating of 6.21 for the happy faces, an average Sadness rating of 4.74 for the sad faces, and an average anger rating of 5.48 for the angry faces. Morph software (Maxwell, 1994) was used to map a set of anchor points onto an image of an actor with a neutral expression onto the same actor expressing an emotion. The Morph program then produced a digital 640- $\times$ 480-pixel movie composed of 100 facial composites such that successive composites changed a mathematically equal degree toward the emotional expression. Thus, for each picture, there was a movie in which the facial expression gradually became more emotional. Each movie was seen twice, and movies were presented in a random order to each participant.

Participants were told they would view movies in which a face initially expressed neutral emotion. They were instructed to slide a bar at the bottom of the screen from left to right, playing each movie at their own speed, and to stop the movie at the first frame where they perceived the face to express an emotion. No verbal labels were provided about which emotion they should expect to see. Participants simply moved a cursor and stopped it at the point where they perceived an emotion expression to emerge on the face. Thus, the principal judgments in this task were nonverbal. Participants clicked the mouse on a button at the bottom of the computer screen to register the frame of expression onset, and a new trial began with a neutral expression. Participants performed two practice trials before beginning the 60 target trials.

\section{Procedure}

Participants visited the laboratory six times during the course of 7 weeks. At the first laboratory session, they completed the personality measures described above, were assigned a palm-top computer (Hewlett Packard $360 \mathrm{LX}$ ), and received instructions for the experience-sampling portion of the study. The experience-sampling procedure lasted 28 days. During this time, an experimenter uploaded participants' emotional experience data to a desktop PC at weekly lab meetings. At each lab meeting, participants were given feedback regarding their level of trial completion (using a companion program called ESPCount; Barrett \& Feldman Barrett, 1999) and also completed a series of laboratory tasks, including the similarity judgment task (Lab Session 2). Approximately 2 weeks after the termination of the experiencesampling period, participants returned to the laboratory for a final time. During that session, they first completed a mood measure and then performed the morph movies task.

The mood measure included adjectives from the Current Mood Questionnaire (Feldman Barrett \& Russell, 1998), allowing us to compute scores for pleasant and unpleasant core affect. Also included

\footnotetext{
${ }^{3}$ The BAS Drive scale (e.g., "I go out of my way to get things I want") and the BAS Fun Seeking scale ("I'm always willing to try something new if I think it will be fun") do not really measure constructs that are relevant to our hypotheses and so were not included in any of the analyses.
} 
were adjectives from the Positive and Negative Affect Schedule-X (Watson \& Clark, 1994), allowing us to compute brief measures of negative and positive activation (NA and PA) as well as scores for momentary Hostility, Sadness, and Joviality. Participants indicated the extent to which affect-related adjectives described their current feelings on 5-point Likert scales $(1=$ not at all, $5=$ very strongly $)$.

\section{Results}

\section{Estimating VF}

We measured VF by computing the proportion of variance in self-reports of emotional experience accounted for by pleasure and displeasure. A brief description of how to compute VF is offered here (for more detail, see Feldman, 1995; Feldman Barrett, 1998).

We computed one $p$-correlation matrix for each person across all experience-sampling measurement instances. This matrix contained the correlations for a set of 16 emotion-related adjectives of interest and represented the relatedness between emotional states for that participant, as reflected in their verbal reporting of internal states. To estimate VF, we quantified the amount of valence-based information contained in each $p$-correlation matrix by comparing it with the valence-based similarity of the words (i.e., similarity in how the words are defined). If, for a given person, there is a correspondence between ratings of emotional experience and the degree of similarity in how the words are defined (in terms of pleasure or displeasure), then this person's ratings contained a lot of valence-based information such that he or she is high in VF. If the valence-based similarity of the words accounts for a small proportion of variance in the ratings of emotional experience, then the person is low in VF.

To estimate the valence-based structure of the words, we computed a valence-based distance matrix. This matrix was derived by subjecting participants' similarity ratings of emotion words to an individualdifferences multidimensional scaling (INDSCAL) analysis using the ALSCAL procedure (Takane, Young, \& DeLeeuw, 1976). A stress-by-dimensions plot for the analysis revealed a clear elbow at the two-dimensional solution, suggesting the suitability of the two-dimensional multidimensional scaling $(\mathrm{MDS})$ solution $\left(\right.$ stress $\left.=.21, R^{2}=.74\right)$. The emotion-related terms fell in a circular order around two dimensions. An inspection of this group solution suggested that one axis represented the valence denoted by the emotion terms, and the other corresponded to the level of arousal. The dimensions represent the agreed-upon attributed or properties of the words (Davison, 1983). Coefficients of congruence with other solutions (depicting a cognitive structure for the same words derived from a different sample of similarity ratings; Feldman, 1995; Feldman Barrett, 2004, Study 2) were above .90 , indicating that the valence-arousal structure replicated at a group level. These findings replicated previous findings that emotion-related adjectives can be necessarily (but not sufficiently) defined in terms of the valence and the level of arousal they denote (Kring, Feldman Barrett, \& Gard, 2003; Russell \& Feldman Barrett, 1999).

The INDSCAL solution provided a definition of each emotion word in terms of its valence and level of arousal. Each word was represented by coordinates on each dimension, indicating its degree of pleasure or displeasure and arousal. From this, we computed a valence-based distance matrix to indicate the proximity of each emotion word to every other in terms of pleasure and displeasure. We computed the valencebased distance matrix for the MDS solution by taking the absolute difference between coordinates for all pairs of emotion words along the valence dimension. Each word had one coordinate on the valence dimension of the solution, yielding 120 valence-based distances. These distances reflected the proximity between every pair of emotion-related words in terms of their pleasure or displeasure. The smaller the absolute value between two coordinates, the smaller the distance between two terms on a dimension, the more similar they are in terms of valence. A similar procedure was followed for coordinates along the arousal dimension.

Each participant's $p$-correlation matrix was correlated with the valence-based distance matrix across the 120 pairs of affect terms, producing a single correlation coefficient that represented the correspondence between how a participant used the adjectives to represent emotional experience and how similar the adjectives were in valence-based meaning. The transformed value of this correlation (by reversing its sign) was an estimate of VF (such that higher values would represent greater focus). We followed a similar procedure to estimate arousal focus (AF) for each person (to be used as a control variable in the final set of analyses). All correlations were subject to Fisher's $r$-to- $z$ transformations before being used in any analyses. 


\section{VF and Perceptual Sensitivity}

To assess whether VF was linked to greater perceptual sensitivity to valenced facial expressions, we began with a multilevel modeling procedure using the Hierarchical Linear Modeling (HLM) program (Raudenbush, Bryk, Cheong, \& Congdon, 2000). Multilevel regression models are used when there are repeated measures (termed Level-1 data) nested within participants (termed Level-2 data). These types of regression models allow investigators to examine the effects of Level-1 and Level-2 variables simultaneously and have several advantages over traditional methods of analyzing repeated measures data (like analysis of variance), including simultaneous estimation of within-subject and between-subject variance, more efficient estimation of effects, lower Type error rates, and no requirement for Level-2 variables to be categorical (Kenny, Korchmarcos, \& Bolger, 2002; Krull \& MacKinnon, 2001). In this study, Level-1 data were the trial-by-trial expression onset judgments for angry, sad, and happy movies. These were nested in participants characterized by differing levels of VF (Level-2 data).

We first conducted one multilevel regression analysis to estimate the average perceived expression onset point for all individuals across all trials $(b=56.52)$, $t(89)=40.96, p<.001$. (Unstandardized regression coefficients are reported in the unit of measurement for the morph movies judgments, which is movie frames.) There was significant variation in participants' average onset times $(u=12.90), \chi^{2}(89)=$ $3045.42, p<.001$, such that some individuals had much earlier onset times than did others. To examine whether individuals with higher VF had earlier onset points than those lower in VF, we included VF as a Level-2 predictor (grand-mean centered). As predicted, VF was related to earlier onset points $(b=$ $-11.38), t(88)=1.65, p<.051$ (one-tailed), following our directional hypothesis.

Next, we explored whether VF moderated the perceived expression onset points for some movies better than for others. We used a multivariate multilevel regression procedure that allowed us to analyze more than one Level-1 criterion variable at a time (e.g., MacCallum, Kim, Malarkey, \& Kiecolt-Glaser, 1997). Here, the onset points for movies of each facial expression (angry, sad, and happy) served as separate Level-1 criterion variables that were included in a single multivariate multilevel regression analysis. This analysis procedure allowed us to simultaneously (a) compute average onset points for each participant for each movie type, (b) determine whether the onset points were related to one another for the three movie types, (c) estimate the variance in the average onset points across participants, and (d) determine the extent to which this variance was predicted by VF.

First, we estimated the average onset points for each type of movie and determined the variance in those averages across individuals (see Table 1). Using multivariate hypothesis testing procedures for fixed effects (Raudenbush et al., 2000), we determined that the average onset points for happy movies, angry movies, and sad movies were all significantly different from one another at $p<.001$. All variances were statistically significant, indicating that participants varied from one another in their onset judgments. Participants' onset points for angry, sad, and happy expressions were highly correlated with one another (correlations ranging from .72 to .97).

Next, we examined the extent to which VF moderated the variability in the Level-1 averages. As predicted, increases in VF were related to significantly earlier perceived onset points for sad facial expressions $(b=-18.74), t(88)=2.45, p<.008$, and for angry facial expressions $(b=-12.95), t(88)=1.80$, $p<.036$ (one-tailed), following our directional hypothesis. Contrary to predictions, VF was not related to earlier onset points for happy facial expressions $(b=-2.47), t(88)=0.29, p<.37$.

\section{Discriminant Validity for the Link Between VF and Perceptual Sensitivity}

To enhance the validity of the VF-perceptual sensitivity link, we examined whether other plausible individual differences (Need For Evaluation, N and E, BIS/BAS), including AF (or the tendency to emphasize felt activation or deactivation in verbal reports of emotional experience) were linked to the point of perceived onset of facial expressions and, if so, whether those differences reduced or eliminated the relation-

Table 1

Descriptive Statistics for Onset Points for All Participants

\begin{tabular}{lccccccc}
\hline & \multicolumn{3}{c}{ Average } & & \multicolumn{3}{c}{ Variability } \\
\cline { 2 - 4 } \cline { 6 - 8 } Movie & $M$ & $t^{\mathrm{a}}$ & $p$ & & $S D$ & $\chi^{2 \mathrm{a}}$ & $p$ \\
\hline Angry & 57.45 & 41.36 & .001 & & 12.78 & 1499.13 & .001 \\
Sad & 66.34 & 44.42 & .001 & & 13.80 & 1732.87 & .001 \\
Happy & 45.77 & 28.83 & .001 & & 14.72 & 1960.53 & .001 \\
\hline
\end{tabular}

Note. $\quad$ Average $=$ grand mean for all participants; metric is in movie frames (possible range is $0-99$ ). Variance $=$ degree to which participants' own average across trials varies from grand mean for all participants.

${ }^{\mathrm{a}} d f=89$. 
ship between VF and earlier onset points. We examined whether the perceived onset of an expression of emotion was influenced by affective state at the beginning of the morph movies task, measured as core feelings of displeasure and pleasure (Feldman Barrett \& Russell, 1998), NA and PA, and discrete feelings of Hostility, Sadness, and Joviality (Watson \& Clark, 1994). Finally, we also examined whether perceived onset of an expression of emotion was influenced by modal affective state, measured as the average experience-sampling ratings for NA and PA, and as ratings of anger, Sadness, and happiness items.

Once again, we performed multivariate multilevel regression analyses. In Step 1 of each analysis, we entered the relevant control variable. The results are presented in Table 2. One multivariate multilevel regression model used AF as the Level-2 predictor for onset judgments for angry, sad, and happy facial expression movies. A second regression used Need For Evaluation as the Level-2 predictor. In the analysis including $\mathrm{N}$ and $\mathrm{E}, \mathrm{N}$ served as the Level-2 predictor for the angry and sad facial expression movies, and $\mathrm{E}$ served as the Level-2 predictor for the happy facial expression movies. We conducted similar analyses for the BIS/BAS Reward Responsiveness subscale (BASReward) measures, for unpleasant and pleasant core affect, and for NA and PA, respectively. In the analyses including ratings of discrete emotional states, ratings of Hostility or anger served as the Level-2 predictor for the angry expression movies, ratings of Sadness served as the Level-2 predictor for the sad expression movies, and ratings of Joviality or happi- ness served as the Level-2 predictor for the happy expression movies. In Step 2 of each analysis, we entered VF as a Level-2 predictor of onset judgments, after the effect of the relevant control variables was accounted for. These results can be found in Table 3 . The relationship between VF and morph movies performance did not change appreciably in any case when control variables were entered into the regression equations first. Thus, none of these variables provided an alternative explanation for the link between VF and onset judgments.

\section{Discussion}

Individuals vary in the extent to which they implicitly focus on feelings of pleasure and displeasure (VF) in reports of emotional experiences. In this study, we showed that this individual difference is related to the perceptual sensitivity to negative information carried in facial expressions of emotion. Individuals high in VF detected the onset of angry and sad facial expressions earlier than did those lower in VF. A similar pattern of results was observed for happy facial expressions, although the effect was not statistically different from chance.

It might be argued that, although we found a link between $\mathrm{VF}$ and performance on the morph movies task, the effect sizes were small, indicating that the effect is not very strong and, by implication, not very important. This is certainly one way to view the findings. Consider what these findings demonstrate, however. They clearly show that information implicitly

Table 2

Step 1 of Discriminant Validity Analyses: Influence of Control Variables on Average Onset Points

\begin{tabular}{lccc}
\hline \multicolumn{1}{c}{ Control variable } & Angry & Sad & Happy \\
\hline Personality characteristic & & & -15.06 \\
$\quad$ Arousal focus & -8.21 & -9.99 & .83 \\
Need for evaluation & 1.76 & 3.63 & $2.24 \dagger$ \\
N/E & 0.08 & 0.14 & 1.29 \\
BIS/BAS & 1.42 & 0.52 & 0.80 \\
Current affect & & & 0.51 \\
$\quad$ Unpleasant/pleasant core affect & -1.25 & -0.82 & 1.80 \\
$\quad$ Negative activation/positive activation & -0.91 & $-1.46 \dagger$ & \\
$\quad$ Hostility/sadness/joviality & & & $2.38 \dagger$ \\
Average experience-sampling rating & -0.83 & -1.99 & 1.64 \\
$\quad$ Negative activation/positive activation & -1.00 & $-1.89 *$ & \\
$\quad$ Anger/sadness/happiness &
\end{tabular}

Note. Metric is in movie frames (possible range $=0-99$ ). Negative values indicate that increases in the control variable were associated with earlier onset frames. $\mathrm{N}=$ neuroticism; $\mathrm{E}=$ extraversion; $\mathrm{BIS}=$ behavioral inhibition system; BAS $=$ behavioral activation system.

$\dagger p<.10 . \quad * p<.05$, two-tailed. 
Table 3

Step 2 of Discriminant Validity Analyses: Effect of VF on Average Onset Points Controlling for the Effect of Control Variables

\begin{tabular}{lccc}
\hline \multicolumn{1}{c}{ Control variable } & Angry & Sad & Happy \\
\hline Original effect of VF on onset points & $-12.95^{*}$ & $-18.74^{* *}$ & -2.47 \\
Personality characteristic & & & \\
$\quad$ Arousal focus & $-16.32^{* *}$ & $-22.77^{* *}$ & -8.63 \\
$\quad$ Need for evaluation & $-12.82^{*}$ & $-18.46^{* *}$ & -2.41 \\
N/E & $-13.54^{*}$ & $-19.64^{* *}$ & -2.36 \\
BIS/BAS & $-13.54^{*}$ & $-19.09^{*}$ & -2.62 \\
Current affect & & & \\
$\quad$ Unpleasant/pleasant core affect & $-17.45^{*}$ & $-18.54^{*}$ & -5.18 \\
$\quad$ Negative activation/positive activation & $-16.54^{*}$ & $-17.49^{*}$ & -5.15 \\
$\quad$ Hostility/sadness/joviality & $-16.12^{*}$ & $-17.11^{*}$ & -5.28 \\
Average experience-sampling rating & & & \\
$\quad$ Negative activation/positive activation & $-12.66^{*}$ & $-17.69^{*}$ & -3.02 \\
Anger/sadness/happiness & $-12.58^{*}$ & $-17.29 *$ & -2.63 \\
\hline
\end{tabular}

Note. Metric is in movie frames (possible range $=0-99$ ). Negative values indicate that increases in valence focus (VF) were associated with earlier onset frames. $\mathrm{N}=$ neuroticism; $\mathrm{E}=$ extraversion; BIS $=$ behavioral inhibition system; BAS = behavioral activation system.

$* p<.05 . \quad * * p<.01$, one-tailed.

contained in self-reports of emotional experience (i.e., VF indices computed on data compiled during the 4 weeks of experience sampling) predict nonverbal, perceptual judgments measured 2 weeks later (after experience sampling had ceased). Viewed from this standpoint, then, our findings offer important convergent validity for the VF construct as well as incremental validity for self-reports of emotional experience, because to our knowledge, this is one of the first articles to show that self-reports of experienced emotion contain implicit information that is meaningful.

Our contention, then, is that our findings provide evidence that the perception of negative cues produces feelings of displeasure, which in turn drives the properties of self-reports (i.e., produces greater emphasis on valence during the self-report process). Of course, we cannot demonstrate these links directly, as there is no way to directly measure what a person is "really" feeling and compare it with his or her report of that experience. We can, however, rule out alternative explanations for observed patterns of behavior. In the present study, we ruled out the possibility that perceptual sensitivity to facial expressions of emotion was due to a host of personality variables and concurrent mood. With these explanations ruled out, we are left with the most plausible explanation for why greater sensitivity to unpleasant cues are related to an emphasis on pleasure and displeasure in self-reports: Greater sensitivity to such cues leads to greater intensity and frequency of unpleasant feelings, which in turn influences how people use emotion adjectives when verbally reporting their feelings.

Moreover, there is additional evidence from research on situated conceptualizations (sometimes called embodied theories) that is consistent with the interpretation that perception causes feeling. According to a situated conceptualization view, people represent knowledge as partial simulations of sensory, motor, and introspective states (Barsalou, 1999; Barsalou, Niedenthal, Barbey, \& Ruppert, 2003; Barsalou, Simmons, Barbey, \& Wilson, 2003). As an event is experienced, the underlying sensory, motor, and introspective states are partially stored. Later, when knowledge of the event becomes relevant in helping to guide perception, these original states are partially simulated. Thus, perceiving an event arises from partially simulating the sensory, motor, and introspective states active at the time. Recent findings (e.g., Adolphs, 2002; Niedenthal et al., 2001) show that people imitate an emotional expression, at least partially, in order to perceive it. By extension, we can hypothesize that people do the same when perceiving the onset of an expression. In the morph movies task, neutral faces gradually and naturally showed increased signs of emotional expression. Once a perceiver registers (even at an unconscious level) evidence of a facial expression, he or she will likely experience some degree of emotional response. Those who more easily detect traces of valenced information in the facial movements of others may imitate these 
movements earlier on during the morph movies task and may also perceive facial expressions of emotion in others with more frequency and variability outside the lab. As a result, they may have more varied affective experiences in everyday life. Of course, this idea must be empirically tested, but we believe that, given the available data, it is a reasonable hypothesis at this point in time.

Finally, demonstrating individual differences in perceptual sensitivity to facial expression is also in and of itself valuable. Individuals were consistent across the three movie types in how early they perceived an emotional expression such that participants with earlier onset times for the angry movies also displayed earlier onset times for the sad and happy movies. Interestingly, the average onset points for the three movie types were all significantly different from one another such that participants perceived happy expressions to emerge earlier than angry expressions, which in turn emerged earlier than sad expressions. This finding may have occurred, in part, because the clarity of the end-point expressions differed across the movie types. Although the original normative ratings of the end-point expressions are no longer available (thus precluding statistical comparisons between them), the end-point expressions for the happy movies were rated as more intensely "happy" than were the end-point angry expressions rated "angry," and both were more intense than were ratings of "sadness" for the sad expression end points. Because each movie frame was a morphed composite of a neutral and an end-point expression, this suggests that the happy-, angry-, and sad-morphed movies contained differing amounts of emotional information at a given frame (e.g., happy movies contained more positive information at Frame 50 than angry or sad movies that contained negative information at that frame). It is not clear whether this difference in end-point intensity influenced the relation between VF and performance on the morph movies task, but it does seem likely that it accounted for differences in average performance across the three movie types. Taken together, this research suggests that, although individuals can classify facial expressions into discrete categories, individuals differ in their perceptions of facial expressions in a more general way, indicating that the encoding of information perceived in the face is far from rigid or inevitable.

\section{References}

Adolphs, R. (2002). Neural systems for the recognizing emotion. Current Opinion in Neurobiology, 2, 69-77.
Barrett, D., \& Feldman Barrett, L. (1999). Experiencesampling program (ESP 2.0). Retrieved from http:// www2.bc.edu/ barretli/esp/

Barsalou, L. W. (1999). Perceptual symbol systems. Behavioral and Brain Sciences, 22, 577-609.

Barsalou, L. W., Niedenthal, P. M., Barbey, A., \& Ruppert, J. (2003). Social embodiment. In B. Ross (Ed.), The psychology of learning and motivation (Vol. 43, pp. 43-92). San Diego, CA: Academic Press.

Barsalou, L. W., Simmons, W. K., Barbey, A. K., \& Wilson, C. D. (2003). Grounding conceptual knowledge in modality-specific systems. Trends in Cognitive Sciences, 7, 84-91.

Carver, C. S., \& White, T. L. (1994). Behavioral inhibition, behavioral activation, and affective responses to impending reward and punishment: The BIS/BAS scales. Journal of Personality and Social Psychology, 67, 319-333.

Davison, M. L. (1983). Multidimensional scaling. Toronto: Wiley.

Feldman, L. A. (1995). Valence focus and arousal focus: Individual differences in the structure of affective experience. Journal of Personality and Social Psychology, 69, 153-166.

Feldman Barrett, L. (1998). Discrete emotions or dimensions? The role of valence focus and arousal focus. Cognition \& Emotion, 12, 579-599.

Feldman Barrett, L. (2004). Feelings or words? Understanding the content in self-report ratings of experienced emotion. Journal of Personality and Social Psychology, 87, 265-281.

Feldman Barrett, L., Quigley, K., Bliss-Moreau, E., \& Aronson, K. (in press). Interoception and self-reports of emotional experience. Journal of Personality and Social Psychology.

Feldman Barrett, L., \& Russell, J. A. (1998). Independence and bipolarity in the structure of current affect. Journal of Personality and Social Psychology, 74, 967-984.

Halberstadt, J. B., \& Niedenthal, P. M. (1997). Emotional state and the use of stimulus dimensions in judgment. Journal of Personality and Social Psychology, 72, 10171033.

Jarvis, W. B. G., \& Petty, R. E. (1996). The need to evaluate. Journal of Personality and Social Psychology, 70, 172-194.

John, O. P., Donahue, E. M., \& Kentle, R. L. (1991). The Big Five Inventory: Technical report. Berkeley: University of California, Institute of Personality and Social Research.

Kenny, D. A., Korchmarcos, J., \& Bolger, N. (2002). Lower-level mediation in multilevel models. Manuscript submitted for publication.

Kring, A. M., Feldman Barrett, L., \& Gard, D. (2003). On 
the broad applicability of the affective circumplex: Representations of affective knowledge among schizophrenia patients. Psychological Science, 14, 207-214.

Krull, J. L., \& MacKinnon, D. P. (2001). Multilevel modeling of individual and group level mediated effects. Multivariate Behavioral Research, 36, 249-277.

MacCallum, R. C., Kim, C., Malarkey, W. B., \& KiecoltGlaser, J. K. (1997). Studying multivariate change using multilevel models and latent curve models. Multivariate Behavioral Research, 32, 215-253.

Maxwell, D. (1994). Morph (Version 2.5) [Computer software]. San Diego, CA: Gryphon Software Corporation.

Niedenthal, P. M., Brauer, M., Halberstadt, J. B., \& InnesKer, A. H. (2001). When did her smile drop? Facial mimicry and the influences of emotional state on the detection of change in emotional expression. Cognition \& Emotion, 15, 853-864.

Niedenthal, P. M., Brauer, M., Robin, L., \& Innes-Ker, Å. (2002). Adult attachment and the perception of facial expression of emotion. Journal of Personality and Social Psychology, 82, 419-433.

Niedenthal, P. M., Halberstadt, J. B., Margolin, J., \& InnesKer, A. H. (2000). Emotional state and the detection of change in facial expression of emotion. European Journal of Social Psychology, 30, 211-222.

Raudenbush, S. W., Bryk, A. S., Cheong, Y. F., \& Congdon, R. (2000). HLM5: Hierarchical linear and nonlinear modeling. Lincolnwood, IL: Scientific Software International.

Russell, J. A., \& Feldman Barrett, L. (1999). Core affect, prototypical emotional episodes, and other things called emotion: Dissecting the elephant. Journal of Personality and Social Psychology, 76, 805-819.

SuperLab. (1999). SuperLab Pro (Version 2.0) [Computer software]. San Pedro, CA: Cedrus Corporation.

Takane, Y., Young, F., \& DeLeeuw, J. (1976). Nonmetric individual differences multidimensional scaling: An alternating least squares method with optimal scaling features. Psychometrika, 42, 7-67.

Watson, D., \& Clark, L. A. (1994). Manual for the Positive Affect and Negative Affect Schedule (Expanded form). Unpublished manuscript, University of Iowa.

Received June 2, 2003 Revision received January 7, 2004 Accepted January 12, 2004

\section{Call for Nominations}

The Publications and Communications (P\&C) Board has opened nominations for the editorships of Clinician's Research Digest, Emotion, JEP: Learning, Memory, and Cognition, Professional Psychology: Research and Practice, and Psychology, Public Policy, and Law for the years 2007-2012. Elizabeth M. Altmaier, PhD; Richard J. Davidson, PhD, and Klaus R. Scherer, PhD; Thomas O. Nelson, PhD; Mary Beth Kenkel, PhD; and Jane Goodman-Delahunty, PhD, respectively, are the incumbent editors.

Candidates should be members of APA and should be available to start receiving manuscripts in early 2006 to prepare for issues published in 2007. Please note that the P\&C Board encourages participation by members of underrepresented groups in the publication process and would particularly welcome such nominees. Self-nominations also are encouraged.

Search chairs have been appointed as follows:

- $\quad$ Clinician's Research Digest: William C. Howell, $\mathrm{PhD}$

- Emotion: David C. Funder, PhD

- JEP: Learning, Memory, and Cognition: Linda P. Spear, $\mathrm{PhD}$, and Peter Ornstein, $\mathrm{PhD}$

- Professional Psychology: Susan H. McDaniel, PhD, and J. Gilbert Benedict, PhD

- Psychology, Public Policy, and Law: Mark Appelbaum, PhD, and Gary R. VandenBos, PhD

Candidates should be nominated by accessing APA's EditorQuest site on the Web. Using your Web browser, go to http://editorquest.apa.org. On the Home menu on the left, find Guests. Next, click on the link "Submit a Nomination," enter your nominee's information, and click "Submit."

Prepared statements of one page or less in support of a nominee can also be submitted by email to Karen Sellman, P\&C Board Search Liaison, at ksellman@ apa.org.

The deadline for accepting nominations is December 10, 2004, when reviews will begin. 